\title{
AVALIAÇÃO TEMPORAL DA LEPTOSPIROSE E OS ÍNDICES DE PRECIPITAÇÃO NAS CAPITAIS DO SUDESTE DO BRASIL
}

Lúcia M. Lobão, Gabriella Bastos, Fernanda Hammer, Gabriel Porto, Ícaro Almeida. Avaliação temporal da leptospirose e os índices de precipitação nas capitais do sudeste do Brasil. Revista Saúde Dinâmica, vol. 9, núm. 3, 2021. Faculdade Dinâmica do Vale do Piranga.

\section{SAÚDE DINÂMICA - Revista Científica Eletrônica FACULDADE DINÂMICA DO VALE DO PIRANGA}

9ạ Edição 2021 | Ano III - no 3 | ISSN - 2675-133X 


\title{
Avaliação temporal da leptospirose e os índices de precipitação nas capitais do sudeste do Brasil
}

\author{
Temporal evaluation of leptospirosis and precipitation indices in southeastern Brazilian \\ capitals \\ Lúcia M. Lobão ${ }^{12}$, Gabriella Bastos ${ }^{3}$, Fernanda Hammer ${ }^{3}$, Gabriel Porto ${ }^{3}$, Ícaro Almeida ${ }^{31}$ \\ ${ }^{1}$ Docente da Escola de Medicina. Faculdade Dinâmica do Vale do Piranga, Ponte nova, Minas Gerais \\ ${ }^{2}$ Docente do Mestrado Profissional em Ensino de Ciências da Saúde e do Ambiente. Faculdade Dinâmica do Vale do \\ Piranga. Ponte Nova, Minas Gerais. \\ ${ }^{3}$ Acadêmicos da Escola de Medicina. Faculdade Dinâmica do Vale do Piranga, Ponte Nova, Minas Gerais \\ Autor correspondente:lucia.fadip@gmail.com
}

\section{Resumo}

O objetivo do presente estudo foi avaliar os casos de leptospirose nas capitais do Sudeste do Brasil, entre 2007 e 2016, associando a fatores como, precipitação mensal, índices de saneamento básico e índices de domicílios em risco de alagamento e rede de esgoto. Os dados foram coletados em diferentes portais governamentais, Sistema de Informações de Notificações e Agravos, Instituto Nacional de Meteorologia, Instituto Brasileiro de Geografia e Estatística e Sistema Nacional de Informações sobre Saneamento. A variação dos dados entre as capitais e os anos foi avaliada através de estatística descritiva. E a relação entre os fatores determinantes e a leptospirose foi estimada através de Correlação de Pearson. A capital com maior média mensal de casos foi São Paulo (19,8 casos). Em todas as capitais, os meses com maiores valores de leptospirose foram dezembro, janeiro, fevereiro e março. Foi encontrada uma relação forte e positiva entre a média de precipitação e as notificações de leptospirose $(r=0,72 ; p<0,05)$. Apesar dos demais fatores apresentarem relações fracas com a leptospirose, é importante destacar que capitais como o Rio de Janeiro apresentam valores altos da doença, apesar do baixo índice pluviométrico quando comparado a outras capitais. Assim, outros fatores como, esgotamento sanitário e quantidade de domicílios em situação de risco poderiam contribuir para o elevado número de casos da doença. Dessa forma, a precipitação mostrou ser um fator explicativo para o número de casos de leptospirose, podendo ser considerado um indicador, que permita a realização de ações que visem a organização do setor de saúde para os possíveis casos de leptospirose.

Palavras-chave: Doença negligenciada; saúde pública, doença infecciosa, leptospirose

\begin{abstract}
The objective of the present study was to evaluate the cases of leptospirosis in the capitals of the Brazilian Southeast, between 2007 and 2016, associating factors such as monthly precipitation, basic sanitation indices and indices of households at risk of flooding and sewerage. Data were collected from different government portals, Sistema de Informações de Notificações e Agravos, Instituto Nacional de Meteorologia, Instituto Brasileiro de Geografia e Estatística and Sistema Nacional de Informações sobre Saneamento. The variation of data between capitals and years was evaluated using descriptive statistics. And the relationship between the determining factors and leptospirosis was estimated using Pearson's correlation. The capital with the highest monthly average of cases was São Paulo (19.8 cases). In all capitals, the months with the highest values of leptospirosis were December, January, February and March. A strong and positive relationship was found between mean precipitation and reports of leptospirosis $(\mathrm{r}=0.72 ; \mathrm{p}<0.05)$. Despite the other factors showing weak relationships with leptospirosis, it is important to highlight that capitals such as Rio de Janeiro have high rates of the disease, despite the low rainfall when compared to other capitals. Thus, other factors such as sanitary sewage and the number of households at risk could contribute to the high number of cases of the disease. Thus, precipitation proved to be an explanatory factor for the number of cases of leptospirosis, and can be considered an indicator that allows actions to be taken to organize the health sector for possible cases of leptospirosis.
\end{abstract}

Key words: Neglected disease; public health, infectious disease, leptospirosis. 


\section{INTRODUÇÃO}

2

3

4

5

6

7

8

9

A leptospirose é uma doença que alcança $40 \%$ de letalidade nos casos graves (MINISTÉRIO DA SAÚDE, 2014), sendo que no período de 2007 a 2017 houve registros de casos de leptospirose em todas as regiões do país, com 42.310 pessoas apresentando diagnóstico confirmado, e uma média anual de 3.846 casos em todo território brasileiro (MARTELI et al., 2020). Ela é uma doença infecciosa febril aguda, causada pela bactéria do gênero Leptospira, sendo caracterizada por um amplo espectro de manifestações clínicas de curso agudo, que inicia após período de incubação de 5 a 14 dias e pode variar desde infecção inaparente até doença fulminante fatal (AROCA et al., 2004).

Entre os sintomas destacam-se febre, mialgia, aparência típica de infecção conjuntival, mialgia intensa que afeta panturrilhas e coxas e limita a marcha, dor retroocular, fotofobia, erupção cutânea petequial no palato, erupção morbiforme não confluente e dor abdominal associada a manifestações digestivas como náusea, vômito e diarréia alta, que às vezes apresenta um diagnóstico diferencial com abdome agudo. Até $10 \%$ dos pacientes evoluem com curso agressivo da doença descrita por icterícia, insuficiência renal aguda, que pode transcorrer com colúria, oligúria ou anúria, e hemorragia, a qual se resulta por, principalmente, sangramento da pele e mucosa, e hemorragia alveolar difusa que ocasiona uma insuficiência respiratória, caracterizandose a Síndrome de Weil (AROCA et al., 2004). O diagnóstico ocorre através da avaliação clínica (observação de sintomas) e laboratorial (testes sorológicos, moleculares e cultura para isolamento da Leptospira) (COELHO et al, 2019).

$\mathrm{Na}$ maioria dos países a leptospirose nos seres humanos é um problema subestimado (HARRISON, 2013). Essa infecção é observada comumente nos trópicos, onde tanto o clima quanto as condições higiênicas, favorecem a sobrevida e distribuição do patógeno (CASTRO et al., 2011). A maior incidência ocorre no verão e outono nos países ocidentais, bem como durante a estação de chuva nos trópicos. A transmissão das leptospiras a seres humanos pode ocorrer após contato direto com a urina, sangue ou tecidos de um animal infectado, ou após a exposição a um ambiente contaminado. A transmissão entre seres humanos é considerada rara (LONG, 2013).

A leptospirose em área urbana, geralmente, está relacionada aos baixos níveis socioeconômicos, sendo que os índices de precipitação pluviométrica precedem surtos aos epidêmicos (GUIMARÃES et al., 2014). Além disso, as condições de saneamento 
básico são determinantes importantes na transmissão dessa doença na área urbana, já que algumas áreas apresentam precariedade das condições ambientais no domicílio e peridomicílio, aliada às altas infestações de roedores, que favorecem o risco de exposição humana (PELISSARI et al, 2011). Assim, a leptospirose tem sido relacionada com atividades ocupacionais e recreacionais, baixas condições de saneamento básico, populações em área de risco para inundações, escoamento das águas pluviais e a densidade demográfica (COSTA et al, 2001).

As epidemias de leptospirose podem resultar da exposição a águas de enchente contaminadas pela urina de animais infectados. Cerca de 102 milhões de pessoas por ano são atingidas por inundações no mundo. No Brasil, as inundações são a segunda maior causa de desastre natural, tendo afetado 28.600 pessoas e causado 6.771 mortes, ambos no período de 1991 a 2010 (GUIMARÃES, 2014). Assim, a ocorrência de Leptospirose está intimamente relacionada com o período chuvoso, pois com o aumento do volume de água, a Leptospira aproxima-se mais rapidamente do homem.

Além disso, é importante ressaltar o fato de que, neste período de clima quente e úmido, as pessoas têm um maior contato com a água de rios, córregos e lagos em atividades de lazer (GUIMARÃES, 2014). Costa et al. (2001) e Gonçalves et al. (2016), através de estudos realizados em cidades brasileiras, destacam a influência das condições ambientais e de fatores socioeconômicos na incidência de leptospirose. Assim, o objetivo do presente estudo foi avaliar o número de casos de leptospirose e a relação com as precipitações pluviométricas nas quatro capitais do sudeste brasileiro, entre os anos de 2007 e 2016. Além disso, comparar essa relação com demais determinantes socioambientais da leptospirose como, densidade demográfica, parcela de domicílios em área de risco, porcentagem da população atendida com abastecimento de água, esgoto e coleta de lixo.

\section{MATERIAIS E MÉTODOS}

A pesquisa trata-se de uma análise ecológica, retrospectiva e observacional realizada nas capitais do sudeste do Brasil, entre o período de 2007 e 2016: Belo Horizonte, Rio de Janeiro, São Paulo e Vitória. O estudo caracteriza-se como descritivo e exploratório, com uma abordagem quantitativa. 
Os dados coletados são referentes aos casos de leptospirose confirmados por mês, sendo estes diagnosticados a partir dos primeiros sintomas e divulgados através do site DATASUS, a partir do SINAN (Sistema Nacional de Agravos de Notificação). Além das incidências de leptospirose, também foram obtidos os valores de precipitações pluviométricas mensais a partir do INMET (Instituto Nacional de Metereologia), dados anuais da população residente nas capitais através do SIDRA pela plataforma do IBGE (Instituto Brasileiro de Geografia e Estatística), dados do número de pessoas atendidas com abastecimento de água, esgotamento sanitário e coleta de resíduos domésticos a partir do SINIS (Sistema Nacional de Informações sobre Saneamento). Todos os valores, anuais ou mensais, foram coletados para o período entre 2007 e 2016. Além disso, foram coletadas informações sobre a densidade demográfica e parcela de domicílios em área de risco de inundação, referente ao ano de 2015, através SINIS. Estes últimos dados foram coletados apenas para o ano citado, pois as informações no sistema iniciaram a partir dessa data, sendo essa coincidente com o período estudado.

As variações dos casos de leptospirose e das precipitações pluviométricas, ao longo de todo período, foram avaliadas através de estatística descritiva, usando histogramas de série de tempo de decomposição. A comparação da variação sazonal, entre os meses, desses dois fatores foi realizada através das médias mensais e desvio padrão, considerando os valores anuais como réplicas no cálculo.

A relação entre os casos de leptospirose e os seus determinantes socioambientais, precipitação, abastecimento de água, esgotamento sanitário e coleta de resíduos sólidos, foi analisada por Correlação de Pearson, considerando um nível de significância de 0,05. Para essa análise, foram calculadas médias anuais dos dados de precipitação e leptospirose, considerando todos os meses no cálculo. A análise foi realizada no software EpiInfo, versão 7.2.2.1.

\section{RESULTADOS E DISCUSSÃO}

\section{Variação das incidências de leptospirose e precipitações pluviométricas}

A análise da distribuição de casos de leptospirose e das precipitações, ao longo do período amostrado, apresentou uma oscilação desses dois parâmetros, ambos com valores elevados em períodos próximos (Figura 1). Observando os intervalos anuais para cada 
98 capital, percebe-se que entre os anos de 2011 a 2013, em Belo Horizonte/MG, houve as 99 maiores notificações da doença, contudo, o índice de chuvas nesses anos não foram os 100 maiores durante o período estudado. Já os maiores valores para o Rio de Janeiro/RJ foram 101 encontrados entre 2008 a 2010, coincidindo com os anos chuvosos nessa região. Para São 102 Paulo/SP, os maiores valores foram encontrados entre 2009 e 2011, sendo o ano de 2009 103 com a maior precipitação. E, finalmente em Vitória/ES ocorreu as maiores incidências 104 entre 2010 a 2011 e um pico em 2014, precedido ao maior valor de precipitação na cidade 105 (713,9mm). 
106

Figura 1: Variação temporal do número de casos de leptospirose (números) e precipitação (mm) entre os anos de 2007 a 2016, nas capitais Belo Horizonte, Rio de Janeiro, São Paulo e Vitória. Os dados de leptospirose foram extraídos do DATASUS, plataforma SINAN e os dados de precipitação do INMET

BELO HORIZONTE

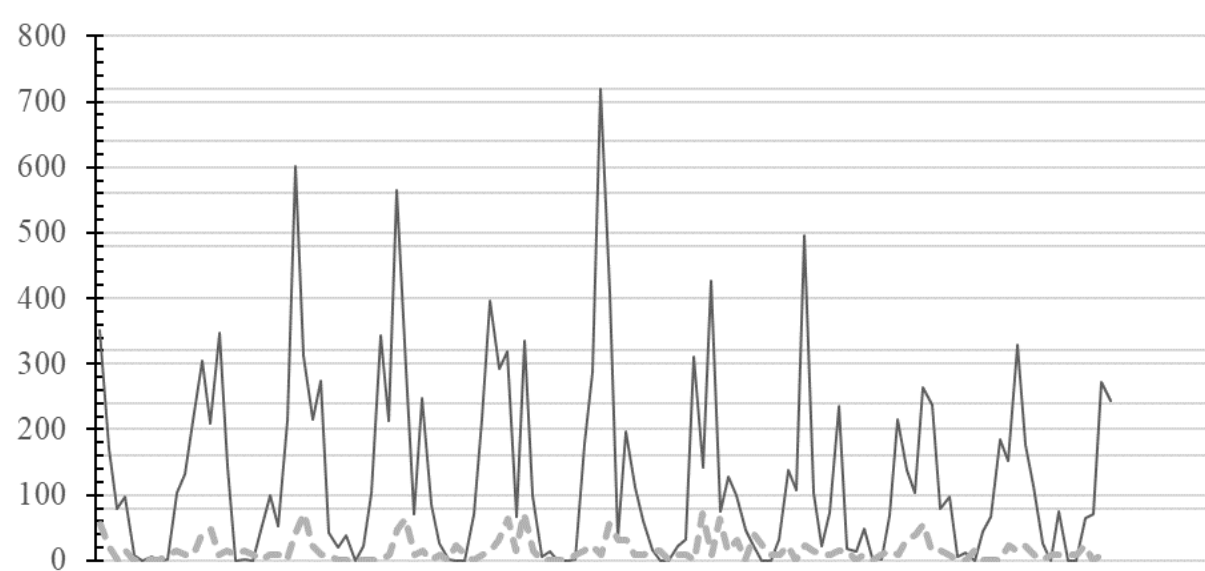

SÃO PAULO

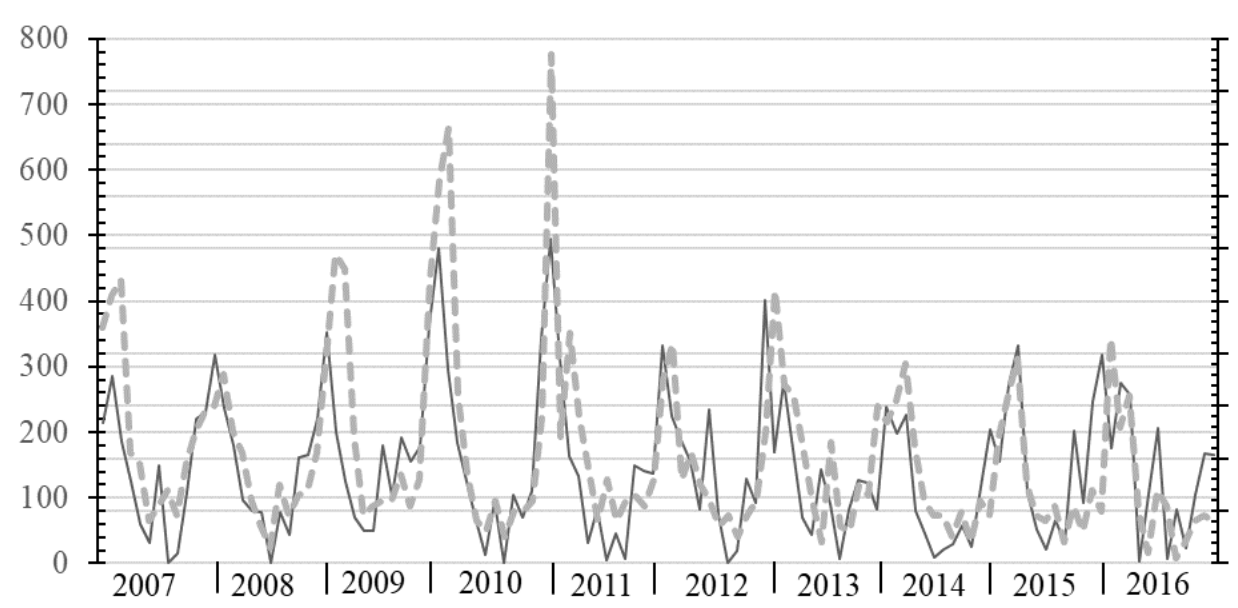

RIO DE JANEIRO

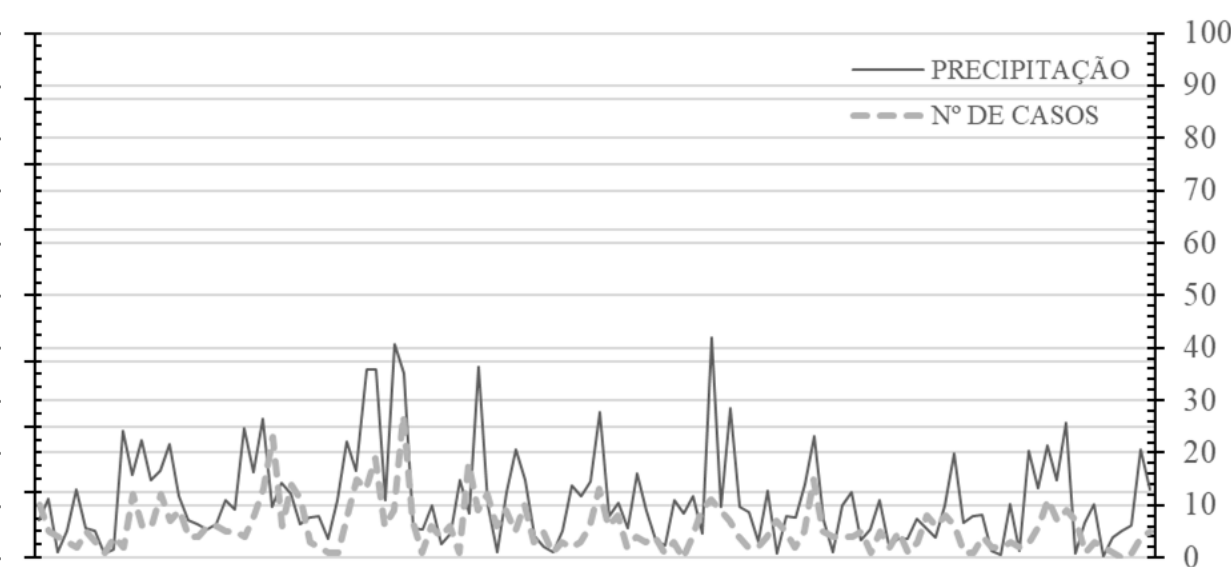

VITÓRIA

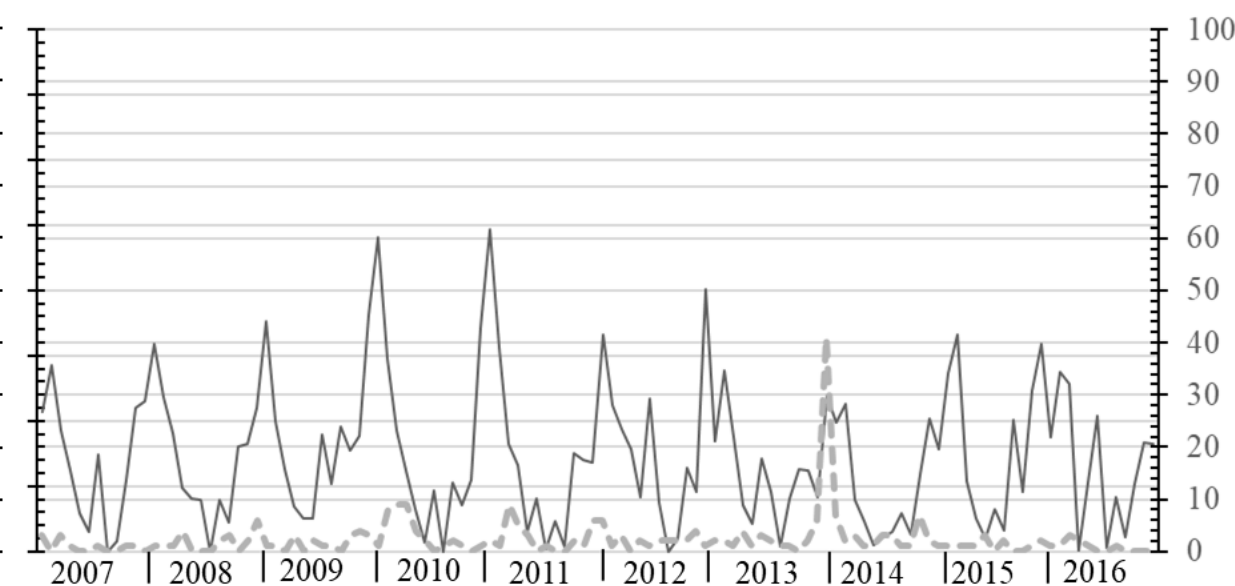


A média dos casos de leptospirose (Tabela 1) e a média do índice de precipitação, para os 10 anos amostrados, mostrou que a capital de São Paulo apresenta os maiores para as duas variáveis, evidenciando a relação entre eles. Entretanto, em Belo Horizonte a média do número de casos é menor e a média de precipitação é maior quando comparado ao Rio de Janeiro. Já Vitória apresenta valores de precipitação semelhantes ao Rio de Janeiro, mas incidências de leptospirose menores (Tabela 1). A comparação das médias entre os meses mostra que, dezembro, janeiro e fevereiro são os meses com número maior de casos de leptospirose

Ressalta-se que o desvio padrão em todos os estados próximos à média, tanto para os casos de leptospirose quanto para precipitação, indicando uma amostra heterogênea com relativa variação entre os anos amostrados (Tabela 1).

Tabela 1: Média e desvio padrão do número de casos de leptospirose (notificações/mês) e precipitação pluviométrica (mm/mês) nas capitais do Sudeste do Brasil: Belo Horizonte, Rio de Janeiro, São Paulo, Vitória. Os valores foram calculados usando os anos como repetições, sendo o período de 2007 a 2016.

\begin{tabular}{|c|c|c|c|c|c|}
\hline \multirow[t]{2}{*}{ CAPITAL } & \multirow[t]{2}{*}{ MESES } & \multicolumn{2}{|c|}{$\mathrm{N}^{\circ}$ DE CASOS/mês } & \multicolumn{2}{|c|}{$\begin{array}{c}\text { PRECIPITAÇÃ̃O } \\
(\mathrm{mm} / \mathrm{mês})\end{array}$} \\
\hline & & Média & Desvio padrão & Média & Desvio padrão \\
\hline \multirow{12}{*}{$\begin{array}{c}\text { Belo } \\
\text { Horizonte }\end{array}$} & JANEIRO & 5,4 & 2,9 & 295,0 & 109,7 \\
\hline & FEVEREIRO & 3,8 & 2,4 & 130,5 & 85,9 \\
\hline & MARÇO & 2,3 & 2,6 & 203,0 & 100,9 \\
\hline & ABRIL & 1,6 & 1,2 & 101,9 & 57,7 \\
\hline & MAIO & 0,7 & 0,7 & 28,3 & 31,3 \\
\hline & JUNHO & 1,0 & 1,6 & 19,0 & 23,1 \\
\hline & \begin{tabular}{|l|} 
JULHO \\
\end{tabular} & 1,1 & 1,2 & 6,7 & 15,1 \\
\hline & AGOSTO & 0,5 & 0,7 & 7,4 & 15,7 \\
\hline & SETEMBRO & 0,9 & 0,9 & 44,3 & 39,1 \\
\hline & OUTUBRO & 1,2 & 1,0 & 127,4 & 96,1 \\
\hline & NOVEMBRO & 0,9 & 1,0 & 233,9 & 85,9 \\
\hline & \begin{tabular}{|l|} 
DEZEMBRO \\
\end{tabular} & 3,7 & 2,5 & 356,4 & 217,7 \\
\hline \multirow{9}{*}{ Rio de Janeiro } & JANEIRO & 10,6 & 4,2 & 156,9 & 105,0 \\
\hline & FEVEREIRO & 8,6 & 5,5 & 74,2 & 40,0 \\
\hline & MARÇO & 6,9 & 1,9 & 147,8 & 90,5 \\
\hline & ABRIL & 7,6 & 7,8 & 96,1 & 77,9 \\
\hline & MAIO & 4,7 & 3,6 & 71,7 & 33,7 \\
\hline & \begin{tabular}{|l|} 
JUNHO \\
\end{tabular} & 2,9 & 1,3 & 51,8 & 17,7 \\
\hline & \begin{tabular}{|l|} 
JULHO \\
\end{tabular} & 3,9 & 1,6 & 47,0 & 34,3 \\
\hline & AGOSTO & 2,6 & 2,3 & 19,5 & 14,7 \\
\hline & SETEMBRO & 3,5 & 1,9 & 56,6 & 27,8 \\
\hline
\end{tabular}




\begin{tabular}{|c|c|c|c|c|c|}
\hline & OUTUBRO & 2,4 & 2,4 & 88,8 & 60,5 \\
\hline & NOVEMBRO & 7,1 & 5,7 & 120,8 & 44,7 \\
\hline & DEZEMBRO & 8,5 & 3,2 & 148,3 & 88,4 \\
\hline \multirow{12}{*}{ São Paulo } & JANEIRO & 46,5 & 123,9 & 293,0 & 123,9 \\
\hline & FEVEREIRO & 41,8 & 18,1 & 257,8 & 40,3 \\
\hline & MARÇO & 36,8 & 12,3 & 201,8 & 57,7 \\
\hline & ABRIL & 20,1 & 5,0 & 96,5 & 43,6 \\
\hline & MAIO & 11,6 & 5,0 & 61,3 & 22,6 \\
\hline & JUNHO & 8,7 & 2,9 & 86,7 & 81,4 \\
\hline & \begin{tabular}{|l|} 
JULHO \\
\end{tabular} & 11,5 & 5,3 & 68,5 & 62,2 \\
\hline & AGOSTO & 8,0 & 4,5 & 38,0 & 38,3 \\
\hline & \begin{tabular}{|l|} 
SETEMBRO \\
\end{tabular} & 9,3 & 3,8 & 74,7 & 71,4 \\
\hline & OUTUBRO & 10,8 & 4,4 & 112,1 & 42,0 \\
\hline & NOVEMBRO & 13,8 & 4,4 & 156,0 & 49,6 \\
\hline & DEZEMBRO & 23,0 & 13,8 & 246,6 & 105,7 \\
\hline \multirow{12}{*}{ Vitória } & JANEIRO & 5,9 & 12,4 & 128,0 & 98,6 \\
\hline & FEVEREIRO & 2,3 & 2,8 & 66,3 & 43,2 \\
\hline & MARÇO & 3,2 & 3,2 & 170,3 & 145,2 \\
\hline & \begin{tabular}{|l|} 
ABRIL \\
\end{tabular} & 3,2 & 2,6 & 116,1 & 112,9 \\
\hline & MAIO & 1,3 & 1,3 & 90,2 & 83,4 \\
\hline & JUNHO & 1,2 & 1,2 & 62,0 & 28,9 \\
\hline & JULHO & 1,0 & 1,1 & 54,8 & 36,9 \\
\hline & AGOSTO & 1,3 & 0,9 & 64,7 & 67,9 \\
\hline & \begin{tabular}{|l|} 
SETEMBRO \\
\end{tabular} & 0,9 & 1,1 & 33,7 & 15,6 \\
\hline & OUTUBRO & 1,0 & 1,1 & 131,0 & 121,7 \\
\hline & NOVEMBRO & 2,2 & 2,2 & 256,8 & 177,6 \\
\hline & DEZEMBRO & 2,7 & 2,5 & 151,0 & 210,4 \\
\hline
\end{tabular}

*Fonte de dados: DATASUS, a partir do SINAN (Sistema Nacional de Agravos de Notificação) e o INMET

O aumento do número de casos de leptospirose ocorre logo após a elevação das precipitações, no mesmo mês ou no seguinte, isso porque a doença possui um período de latência que pode variar de 3 a 30 dias, com uma média de 10 dias (HARRISON, 2013), influenciando diretamente sobre o diagnóstico de novos casos. É possível inferir, portanto, que a ocorrência de leptospirose está estreitamente relacionada com o elo hídrico predominante no verão, pois, com o aumento do volume de água e também formação de lama, a Leptospira é transportada de forma mais eficaz através das águas de inundações (GONÇALVEZ et al, 2016), confirmando os dados encontrados no presente estudo. 
Segundo Guimarães et al. (2014), um fator potencializador de epidemias são as fortes chuvas sazonais em regiões tropicais, que ao causarem enchentes e inundações, tornam-se a condição favorável para a exposição ao agente da doença por meio da transmissão hídrica em áreas urbanas. Gonçalves et al. (2016) encontraram uma associação direta entre a incidência da doença e a variação pluviométrica, mostrando que, nos anos com os maiores índices pluviométricos ocorreu um aumento do número de casos confirmados de leptospirose. É importante frisar também, que a pluviosidade e o número de casos de leptospirose têm relação direta com a saturação do solo e a urbanização. Outro fator que contribui para essa relação é que nas épocas de enchentes, já são esperados os casos de leptospirose, sendo assim há maior número de diagnósticos preliminares corretos e de solicitações de exames. Nos anos em que não há inundações, o diagnóstico é feito tardiamente e aumentam os números de óbitos (KUPEK e FAVERSANI, 2000).

\section{Relação entre a leptospirose e seus determinantes socioambientais}

A densidade demográfica, a rede de abastecimento de água, rede de esgoto e coleta de lixo, assim como a porcentagem de moradias em risco de inundação, também são elementos que aumentam a probabilidade de incidência da leptospirose. Os valores apresentados por essas variáveis nas capitais representam uma tendência de associação entre eles e o risco de ocorrência das infecções por Leptospira.

A tabela 2 aponta que as capitais com maiores densidades demográficas são Rio de Janeiro e São Paulo, seguida de Belo Horizonte e Vitória. Quase 100 \% da população, em todas as capitais possuem abastecimento de água e coleta de resíduos sólidos. Contudo, existem diferenças na parcela da população em situação de risco e que possui esgotamento sanitário (Tabela 2). Rio de Janeiro se destaca com a maior parcela de domicílios em área de risco de inundação (aproximadamente 20\%), seguido de Belo Horizonte, São Paulo e Vitória. Em relação ao esgotamento sanitário, Vitória possui pouco mais $60 \%$ da população atendida, Rio de Janeiro 79\%, enquanto que Belo Horizonte e São Paulo possuem mais de $90 \%$ da população atendida.

Barcellos e Sabroza (2000), identificaram em seu estudo, que as condições de saneamento e dos riscos de inundações foram apontadas como as principais variáveis que determinam os casos de leptospirose. Teixeira e seus colaboradores (2018) demostram que há, nas regiões 
metropolitanas dos grandes centros, uma alta densidade demográfica e uma grande produção de resíduos sólidos. Esses fatores associados a precariedade do saneamento básico, além do alto índice de área construída, acarretam na impermeabilização do solo urbano e propiciam o escoamento superficial, levando a maior ocorrência de enchentes. Assim, essas populações apresentam maior risco de adquirir a infecção (TEIXEIRA et al. 2018). Fatores como domicílios em área de risco e saneamento básico, refletem na quantidade de roedores no peridomicílio e, consequentemente, no número de casos da doença (BARCELLOS; SABROZA, 2000).

Tabela 2: Capitais e fatores de risco associados aos casos de leptospirose, estimados em habitantes por quilômetro quadrado e porcentagem (\%): densidade demográfica, abastecimento de água, rede de esgoto, domicílio de risco, canais de águas pluviais.

\begin{tabular}{|c|c|c|c|c|c|c|}
\hline \multirow[b]{2}{*}{ Capital } & \multirow[b]{2}{*}{$\begin{array}{c}\text { População } \\
\text { residente } \\
\text { (habitantes) }\end{array}$} & \multirow[b]{2}{*}{$\begin{array}{c}\text { Densidade } \\
\text { demográfica } \\
\text { pessoa/ha* }\end{array}$} & \multirow{2}{*}{$\begin{array}{c}\text { Domicílios } \\
\text { em Situação } \\
\text { de Risco de } \\
\text { Inundação } \\
\text { (\%) }\end{array}$} & \multicolumn{3}{|c|}{ \% da população atendida } \\
\hline & & & & $\begin{array}{c}\text { Abastecimento } \\
\text { de água }\end{array}$ & Esgoto & $\begin{array}{c}\text { Coleta de } \\
\text { lixo }\end{array}$ \\
\hline $\begin{array}{l}\text { Belo } \\
\text { Horizonte }\end{array}$ & 2.450 .824 & 76 & 2,3 & 100 & 98 & 96 \\
\hline $\begin{array}{l}\text { Rio de } \\
\text { Janeiro }\end{array}$ & 6.341 .080 & 112 & 19,8 & 95 & 79 & 100 \\
\hline São Paulo & 11.495 .680 & 110 & 1,6 & 99 & 94 & 99 \\
\hline Vitória & 337.172 & 37 & 0,4 & 99 & 62 & 100 \\
\hline
\end{tabular}

*Fonte: SIDRA pela plataforma do IBGE (Instituto Brasileiro de Geografia e Estatística) e SINIS (Sistema Nacional de Informações sobre Saneamento)

A análise de correlação demonstrou que os índices de precipitação são determinantes para a incidência de leptospirose nas capitais ( $\mathrm{r}=0,72$, Tabela 3). Os demais determinantes apresentaram uma relação considerada fraca (abastecimento de água $r=0,04$; coleta de lixo $r=$ 0,12), ou não significativa, como no caso da parcela de domicílios em área de risco (p>0,05, valor do coeficiente de Pearson não inserido na tabela). Contudo, é importante destacar que, alguns valores altos do número de casos de leptospirose ocorrem em capitais com baixa precipitação, mas com grande parcela da população em área de risco e com condições de saneamento mais precária quando comparada a outras capitais. 
Tabela 3: Correlação de Pearson entre o número de casos de leptospirose e seus determinantes socioambientais. O nível de significância considerado foi $\mathrm{p}<0,05$. Foram apresentados apenas correlações significativas.

\begin{tabular}{c|c|c|c}
\hline \multirow{2}{*}{ Determinantes } & \multicolumn{2}{|c}{ Casos de leptospirose } \\
\cline { 2 - 4 } & Coeficiente de Pearson (r) & $p$ & $\mathrm{~N}$ \\
\hline Precipitação & 0,72 & $<0,05$ & 39 \\
\hline Abastecimento de água & 0,04 & $<0,05$ & 35 \\
\hline Esgotameneto sanitário & 0,39 & $<0,05$ & 35 \\
\hline Coleta de lixo & 0,12 & $<0,05$ & 35 \\
\hline
\end{tabular}

No Brasil, a leptospirose ainda é um grande problema de saúde pública, e isso se deve a vários fatores como: alterações climáticas, transição demográfica com formação de favela, desenvolvimento de comunidades sem saneamento básico, esgoto, água potável, coleta de lixo, além do baixo nível de educação, pouco acesso à informação e, principalmente, falta de decisão política, evidenciando assim, que a leptospirose é uma doença que afeta quase que prioritariamente a população negligenciada (SBMT, 2014) .

As áreas de favelas e as áreas semirrurais, além das periféricas montanhosas, possuem dificuldades no acesso básico de infraestrutura sanitária e por isso, restrições severas para coleta de resíduos, tratamento de esgoto e abastecimento de água, o que pode favorecer a distribuição das leptospiras através do meio hídrico e solos contaminados com as leptospiras (SOARES, 2010).

A Organização Mundial de Saúde (OMS) estima que mais de um bilhão de pessoas estão infectadas com pelo menos uma doença negligenciada e, apesar de potencialmente fatais, Halliday et al (2015) afirmam que justamente por englobarem populações em condições precárias e não promoverem um retorno financeiro significativo, não despertam o interesse da indústria farmacêutica ou estudos de empresas privadas, tendo seus investimentos exclusivamente públicos. As doenças negligenciadas são doenças transmitidas por agentes infecciosos que atingem desproporcionalmente as populações de baixa renda, que vivem em condições precárias de higiene, sem saneamento básico adequado ocorrendo principalmente na África, Ásia e América Latina (MINISTÉRIO DA SAÚDE, 2010). Dessa forma, os índices populacionais de atingidos pela leptospirose continuam sendo altos e atingem regiões com condições mais precárias.

\section{CONCLUSÃO}


Os dados levantados nesse estudo confirmam que a leptospirose ainda é uma doença presente na população e que está associada às questões ambientais e às condições sociais. Tal fato demonstra a necessidade de investimento nas condições básicas de saneamento para melhoria da saúde populacional. Enquanto não houver investimento em qualidade de vida e estrutura urbana, doenças como a leptospirose, continuarão presentes na população e contribuindo para o aumento do número de óbitos. É provável que os indivíduos vacinados possam mudar seu comportamento e diminuir a adesão às medidas de prevenção (por exemplo, distanciamento físico, máscaras faciais) o que pode aumentar a transmissão viral e retardar o final desse pesadelo chamado Pandemia. Apesar disso, acreditamos que nossas descobertas têm importantes implicações para a saúde, principalmente no intuito de mostrar ao público sobre a necessidade e o benefício das campanhas de vacinação.

\section{REFERÊNCIAS}

AROCA, G.; ACCINI, J.L.; PÉREZ, R.; RODELO, E.; DAU, H. Leptospirosis ictérica: Síndrome de Weil's. Salud Uninorte, (19): 31-40, 2004. Disponível em: https://www.scielo.br/pdf/rsbmt/v3n2/a04v3n2.pdf.

BARCELLOS, C; SABROZA, P. Socio-environmental determinants of the leptospirosis outbreak of 1996 in western Rio de Janeiro: a geographical appoach. Intern J of Environ Health Res, San Diego, n10 (4): 301-313, 2000. Disponível em: https://www.arca.fiocruz.br/handle/icict/542.

CARVALHO, C.R.R.; BETHLEM, E.P. Complicações pulmonares da leptospirose. Clin Chest Med. n23: 469-78, 2002. Disponível em: https://www.scielo.br/pdf/jpneu/v28n4/12967.pdf

CASTRO, J.R; SALABERRY, S.R.S; SOUZA M.A; LIMA-RIBEIRO, A.M.C. Sorovares de Leptospira spp. predominantes em exames sorológicos de caninos e humanos no município de Uberlândia, Estado de Minas Gerais. Rev. Soc. Bras. Med. Trop. n44(2): 217-222, 2011. Disponível em: http://www.scielo.br/scielo.php?script=sci_arttext\&pid=S0037$86822011000200018 \& \operatorname{lng}=$ en

COELHO, A; ALVES, I; FARIAS V. Perfil epidemiológico dos casos de leptospirose na Região Metropolitana da Baixada Santista (SP), Brasil. BEPA. n16 (183): 3-14, 2019. Disponível em: http://docs.bvsalud.org/biblioref/2019/10/1023295/151833-14.pdf.

COSTA, E; COSTA, Y.A; LOPES, A.A; SACRAMENTO E; BINA J.C. Formas graves de leptospirose: aspectos clínicos, demográficos e ambientais. Rev. Soc. Bras. Med. Trop. n34(3): 261-267, 2001. Disponível em: 
http://www.scielo.br/scielo.php?script=sci_arttext\&pid=S0037-

$86822001000300006 \& \operatorname{lng}=$ en.

DOLHNIKOFF, M; MAUAD, T; BETHLEM, E.P; CARVALHO, C.R.R. Patologia e fisiopatologia das manifestações pulmonares na leptospirose. Rev. Bras. Doenças Infec. n11 (1): 142-148, 2007.

FILHO, M.R.; CAVALHEIRO, C.S.; PEDROSO, J.V.M. Hemorragia pulmonar resultante de leptospirose. Fac Ciênc Méd Sorocaba, 18(2): 117-20, 2016. Disponível em: https://pdfs.semanticscholar.org/7f4d/1b8bd2c039f9df3c8861f84a158eb9b7d898.pdf.

GONÇALVES, N.V; ARAÚJO, E.N; SOUSA, J.A.S; PEREIRA, W.M.M; MIRANDA, C.S.C; CAMPOS, P.S.S; Matos, M.W.S; PALÁCIOS, V.R.C.M. Distribuição espaçotemporal da leptospirose e fatores de risco em Belém, Pará, Brasil. Ciênc. Saúde Coletiva, n21(12): 3947-3955, 2016. Disponível em:

http://www.scielo.br/scielo.php?script=sci_arttext\&pid=S1413$81232016001203947 \& \operatorname{lng}=$ en.

GUIMARÃES, R.M; CRUZ, O.G; PARREIRA, V.G; MAZOTO, M.L; VIEIRA, J.D; ASMUS, C.I.R.F. Análise temporal da relação entre leptospirose e ocorrência de inundações por chuvas no município do Rio de Janeiro, Brasil, 2007-2012. Ciênc. Saúde Coletiva, n19(9): 3683-3692, 2014. Disponível em:

http://www.scielo.br/scielo.php?script=sci_arttext\&pid=S1413$81232014000903683 \& \operatorname{lng}=$ en.

HALLIDAY, J.E; ALLAN, K.J; EKWEM, D; CLEAVELAND, S; KAZWALA, R.R; CRUMP, J.A. Endemic zoonoses in the tropics: a public health problem hiding in plain sight. The Veterinary record, n176(9), 220-225, 2015. Disponível em:

https://veterinaryrecord.bmj.com/content/176/9/220

IBGE (Instituto Brasileiro de Geografia e Estatística). Cidades e Estados. 2010 Disponível em: https://www.ibge.gov.br/cidades-e-estados. Acesso em: 20 de fevereiro de 2020.

INMET (Instituto Nacional de meteorologia. Precipitação). Disponível em: http://www.inmet.gov.br/portal/. Acesso em: 20 de fevereiro de 2020.

KUPEK, E; FAVERSANI, M.C. The relationship between rainfall and human leptospirosis in Florianópolis, Brazil, 1991-1996. Brazil J Infect Dis, n4 (3): 131-134, 2000. Disponível em: https://pubmed.ncbi.nlm.nih.gov/10934496/.

LONG, Dan L. et al. Medicina Interna de Harrison. 18 ed. Porto Alegre, RS: AMGH Ed., 2013. p.1048-1051.

MELO, T. F; PECONICK, A. P. As características da Leptospira spp.:uma revisão de literatura. Scire Salutis, v.9, n.3, p.1-7, 2019. Disponível em:

file://C:/Users/User/Downloads/3494-Texto\%20do\%20artigo-7835-1-1020191112\%20(1).pdf.

MINISTÉRIO DA SAÚDE (BR). Doenças negligenciadas: estratégias do Ministério da Saúde. Rev. Saúde Pública, n44(1): 200-2, 2010. 
MINISTÉRIO DA SAÚDE. Secretaria de Vigilância em Saúde. Departamento de Vigilância das Doenças Transmissíveis. Leptospirose: diagnóstico e manejo clínico. Brasília :

Ministério da Saúde, 2014. Disponível em:

http://bvsms.saude.gov.br/bvs/publicacoes/leptospirose-diagnostico-manejo-clinico2.pdf.

PELISSARI, D.M; MAIA-ELKHOURY, A.N.S; ARSKY, M.L.N.S; NUNES, M.L. Revisão sistemática dos fatores associados à leptospirose no Brasil, 2000-2009. Epidemiol. Serv. Saúde, n20(4): 565-574, 2011. Disponível em:

http://scielo.iec.gov.br/scielo.php?script=sci_arttext\&pid=S167949742011000400016\&lng= pt.

PICARDEAU, M. Diagnosis and epidemiology of leptospirosis. Med Mal Infect, n43, 1-9, 2013. Disponível em:

https://www.sciencedirect.com/science/article/pii/S0399077X12003198?via\%3Dihub\#bibl0 005 .

SINAN: Sistema de Informação de Agravos de Notificação. 2019. Disponível em: http://dtr2019.saude.gov.br/sinanweb/novo/Documentos/SinanNet/leptospirose.pdf. Acesso em: 21 de outubro de 2019.

SISTEMA NACIONAL DE INFORMAÇÕES SOBRE SANEAMENTO (SNIS) 2019. Disponível em: http://appsnis.mdr.gov.br/indicadores/web/agua_esgoto/mapa-agua. Acesso em: 21 de outubro de 2019.

SOARES, T.S.P; LATORRE, M.R.D.O; LAPORTA, G.Z; BUZZAR, M.R. Análise espacial e sazonal da leptospirose no município de São Paulo, SP, 1998 a 2006. Rev Saude Publica, n44(2): 687-694, 2010. Disponível em:

http://www.scielo.br/scielo.php?script=sci_arttext\&pid=S0034-

$89102010000200008 \& \operatorname{lng}=\mathrm{en}$.

SOCIEDADE BRASILEIRA DE MEDICINA TROPICAL. Falta de decisão política contribua para ocorrência de leptospirose. 2014. Disponível em:

https://www.sbmt.org.br/portal/falta-de-decisao-politica-contribui-para-ocorrencia-de-

leptospirose/.

\section{Declaração de Interesse}

Os autores declaram não haver nenhum

conflito de interesse

\section{Financiamento}

Financiamento próprio

\section{Agradecimentos}

Agradecemos ao suporte fornecido pela Coordenação de Medicina da Faculdade

Dinâmica do Vale do Piranga

\section{Colaboração entre autores}

O presente artigo foi escrito pelos discentes G.B.; F.H.; G.P.; I.A. . sob orientação da professora L.M.L.., projetado e concluído pela disciplina Atividades complementares da Escola de Medicina, Faculdade Dinâmica do Vale do Piranga.

Os autores participaram de todas as etapas. 